\title{
Theoretical investigation of bonding in diaspore
}

\author{
BJÖRN WINKLER ${ }^{1 *}$, MAREK HYTHA ${ }^{1}$, CHRIS PICKARD ${ }^{1}$, VICTOR MILMAN², \\ MICHELE WARREN ${ }^{3}$, MATTHEW SEGALL ${ }^{4}$
}

\author{
${ }^{1}$ Kristallographie / Institut für Geowissenschaften, Olshausenstr. 40, D-24098 Kiel, Germany \\ * corresponding author. E-mail: bjoern@min.uni-kiel.de \\ ${ }^{2}$ Molecular Simulations Inc., The Quorum, Barnwell Road, Cambridge, CB5 8RE, UK \\ ${ }^{3}$ Dept of Earth Sciences, University of Manchester, Oxford Road, Manchester, M13 9PL, UK \\ ${ }^{4}$ TCM Group, Cavendish Laboratory, Madingley Road, Cambridge, CB3 0HE, UK
}

\begin{abstract}
The bulk modulus of diaspore, $\boldsymbol{\alpha}-\mathrm{AlOOH}$, has been obtained from density functional theory based calculations. The value obtained, $B=148 \mathrm{GPa}$, is consistent with that previously obtained from elastic constant measurements, but in strong disagreement with values derived from high pressure x-ray diffraction experiments. A Mulliken bond population analysis of the electronic structure implies that the Al-O bonds are significantly covalent, in contrast to findings based on an earlier x-ray diffraction study. On compression, the main change is the increase in the hydrogen-bond strength.
\end{abstract}

Key-words: diaspore, $\mathrm{AlOOH}$, high pressure, density functional theory.

\section{Introduction}

The stability field and the physical properties of diaspore, $\boldsymbol{\alpha}-\mathrm{AlOOH}$, are of interest, as this phase may play a role as a source of $\mathrm{H}_{2} \mathrm{O}$ in subducting slabs and its properties are required for petrogenetic modelling of some reactions in the $\mathrm{CASH}-$ and MASH-system. From a crystallographic perspective, diaspore is an interesting model system to study hydrogen bonds of intermediate strength as its structure is comparatively small and simple. $\boldsymbol{\alpha}$ AlOOH crystallizes in space group Pbnm with $Z=4$. The structure can be described in terms of a slightly distorted hexagonally close packed arrangement of oxygens, with Al-atoms occupying some of the octahedral interstices (Table 1). The nonlinear hydrogen bond, -Al-O(2)-H...O(1)-Al-, is known to be of intermediate strength, as the $\mathrm{O}-\mathrm{H}$ stretching frequency has a value of about $3000 \mathrm{~cm}^{-1}$ (Ryskin, 1974).

Due to its petrological importance, the stability of diaspore as a function of pressure and temperature has been investigated extensively (e.g. Pawley et al. (1996); Holland et al. (1996); Fockenberg et al. (1996) and references therein). However, experimentally determined values for the bulk modulus, $B$, differ significantly. A value $B=230 \mathrm{GPa}$ has been obtained from energy dispersive $\mathrm{x}$-ray diffraction experiments (Xu et al., 1994). Mao et al. (1994) obtained a bulk modulus of $B=167.5 \mathrm{GPa}$. Fasshauer et al. (1998) use a value of $B=134 \mathrm{GPa}$. An earlier study came to the conclusion that $B$ is even lower and gave $B=85 \mathrm{GPa}$ (Ruoff \& Vanderborgh, 1991). The elastic, $c_{i j}$, and thermoelastic, $T_{i j}$, constants have also been determined (Haussühl, 1993). The $c_{i j}$ are given in Table 2 . The bulk modulus calculated from the elastic constants at room temperature is $147 \mathrm{GPa}$. An extrapolation of the experimental $c_{i j}$ to $0 \mathrm{~K}$ gives $B=152 \mathrm{GPa}$. Clearly, there is a need to clarify the value of $B$.

The electron density distribution at ambient conditions has been determined by x-ray diffraction (Hill, 1979). However, features in the resultant deformation maps are difficult to analyze due to the presence of many local maxima and minima. No significant charge accumulation between the Al- 
atoms and the neighboring $\mathrm{O}(1)$ and $\mathrm{O}(2)$ atoms was detected, and it was therefore concluded that the bonding is primarily ionic. From a conventional crystal chemical viewpoint, this is surprising as the Pauling electronegativities of $\mathrm{Al}$ and $\mathrm{O}$ differ by 2.0 , which is usually taken as an indication that an $\mathrm{Al}-\mathrm{O}$ bond is appreciably covalent in character.

In addition to further constraints on $B$ and a reinvestigation of the charge distribution, what has been missing up to now is a comprehensive discussion of pressure-induced structural changes and their effects on the nature of the interatomic bonds. This would help interpret the conflicting results concerning the elastic properties, clarify the influence of the hydrogen bond on structural properties and would be of general relevance for an understanding of properties of the many isotypic structures. Such a study is best done with a parameter-free atomistic model, as from such a model the elastic constants, compression behavior, bond population and pressure-induced structural changes can be obtained simultaneously. Such model calculations, which we present here, are therefore ideally suited to complement the existing experimental data.

For crystals, most quantum mechanical calculations are currently based on density functional theory (DFT) (Hohenberg \& Kohn, 1964; Kohn \& Sham, 1965; Parr \& Yang, 1989; Kryachko \& Ludena, 1990; Jones \& Gunnarsson, 1989). While DFT itself is exact (Hohenberg \& Kohn, 1964), practical calculations require an approximation for the treatment of the exchange and correlation energies. Here we use the 'generalized gradient approximation', in the form suggested by Perdew, Burke and Ernzerhof, GGA-PBE (Perdew et al., 1996). Results based on GGA calculations are generally in better agreement with experiment than those obtained with the local density approximation, LDA (Leung et al., 1991; Hammer et al., 1993; Goniakowski et al., 1996; Hamann, 1996). The study of structures with large unit cells or low symmetries requires a computationally efficient approach. Currently, computational schemes in which the charge density and electronic wavefunctions are expanded in a basis set of plane waves are often used. However, as it is impractical to consider tightly bound core electrons explicitly when using a plane-wave basis set, pseudopotentials have to be introduced to mimic the screening of the Coulomb potential of the nucleus by the core electrons. A number of approaches for the construction of pseudopotentials have been presented in the literature (Bachelet et al., 1982; Kleinman \& Bylander, 1982). The stateof-the-art are the efficient 'ultrasoft' pseudopoten- tials, which require a comparatively small number of plane waves (Vanderbilt, 1990; Kresse \& Hafner, 1994).

While a DFT-based plane-wave/ultrasoft pseudopotential approach is therefore well-suited to study complex crystal structures, this efficient computational scheme lacks one appealing property of models based on localized basis sets, namely the possibility to express the computed charge density in terms of the familiar atomic orbitals and compute partial charges and bond populations, as is commonly done in quantum chemistry. It is generally acknowledged that such a 'Mulliken analysis' only yields semi-quantitative information, as the underlying crystal chemical concepts do not correspond to quantum mechanical observables. However, the relative magnitude of bond populations and changes due to variations of the structural parameters, for example induced by increasing pressure, can be monitored. To obtain the same information available from computations based on a localized basis set, while maintaining the efficiency and flexibility of a plane wave calculation, a recently developed method to project the computed electron density on a localized basis set (Sanchez-Portal et al., 1996) can be used.

While the bulk modulus, $B$, and its pressure derivative $B^{\prime}$ can be obtained in a rather straightforward manner in DFT-calculations by fitting an equation of state to unit cell volumes obtained as a function of pressure, the computation of elastic constants is more involved. The most elegant method is based on "linear response" (perturbation-) theory. However, this method has not yet been developed for ultrasoft pseudopotentials. What is used here instead is an approach based on the imposition of strain patterns on a structure and the computation of the resultant stress tensor. This then allows, via Hooke's law, the derivation of elastic constants for complex, low symmetry structures with an affordable computational effort.

\section{Computational details}

The quantum mechanical calculations performed here are based on density functional theory, DFT, and the PBE-version of the GGA. Ultrasoft pseudopotentials were used with a maximum cutoff energy of the plane waves of $380 \mathrm{eV}$. In addition to the cutoff energy, only one further parameter determines the quality of the calculations, namely the density of points with which the Brillouin zone is sampled. The wave vectors for the sampling points 
were chosen according to the scheme proposed by Monkhorst \& Pack (1976). Here, we use a sampling of reciprocal space such that distances between grid points are about $0.05 \AA^{-1}$. Full geometry optimization calculations were performed, i.e. all structural parameters not constrained by the space group symmetry were relaxed. After the final selfconsistency cycle, the remaining forces on the atoms were less than $0.02 \mathrm{eV} / \AA$, and the remaining stress was less than $0.1 \mathrm{GPa}$. The present calculations are restricted to the athermal limit, in which temperature effects and zero-point motions are neglected. For all calculations we used academic and commercial versions of the CASTEP program, which has been described elsewhere (Payne et al., 1992; MSI, 1998; Milman et al., 2000). The implementation of the population analysis is that of Segall et al. (1996). The elastic constants calculations were performed by imposing strains on the relaxed structures with subsequent optimization of all internal parameters. In the present case, three strain patterns are required to obtain all elastic constants. The magnitude of the applied distortion is varied over a small range (up to a relative change of $0.2 \%$

Table 1. Structural parameters of diaspore at 0 and $50 \mathrm{GPa}$. The experimentally determined fractional coordinates are from Busing \& Levy (1958), the lattice parameter have been taken from Fockenberg et al. (1996). $\alpha$ is the $\mathrm{H}-\mathrm{O}(2)-\mathrm{O}(1)$ angle. ${ }^{1}$ exp. data from Ruoff \& Vanderborgh (1991); Xu et al. (1994); Mao et al. (1994). ${ }^{2}$ exp. data as cited by Fasshauer et al. (1998). ${ }^{3}$ theoretical data from equation of state,${ }^{4}$ theoretical data from strain calculations. The errors associated with the theoretical bulk moduli are derived from the fit of the equation of state to the $P-V$ data and from the uncertainties of the theoretical elastic constants.

\begin{tabular}{llll}
\hline & exp. & $\begin{array}{l}\text { theo. } \\
0 \mathrm{GPa}\end{array}$ & $\begin{array}{l}\text { theo. } \\
50 \mathrm{GPa}\end{array}$ \\
\hline$a[\AA]$ & $4.3995(6)$ & 4.377 & 4.017 \\
$b[\AA]$ & $9.427(2)$ & 9.341 & 8.812 \\
$c[\AA]$ & $2.8442(5)$ & 2.816 & 2.638 \\
$\mathrm{~A} 1(\mathrm{x})$ & $0.0451(8)$ & 0.0462 & 0.0327 \\
$\mathrm{Al}(\mathrm{y})$ & $0.8554(3)$ & 0.8558 & 0.8627 \\
$\mathrm{O} 1(\mathrm{x})$ & $0.7120(5)$ & 0.7112 & 0.7101 \\
$\mathrm{Ol}(\mathrm{y})$ & $0.1989(2)$ & 0.1988 & 0.1978 \\
$\mathrm{O} 2(\mathrm{x})$ & $0.1970(5)$ & 0.1954 & 0.2198 \\
$\mathrm{O} 2(\mathrm{y})$ & $0.0532(2)$ & 0.0544 & 0.0510 \\
$\mathrm{H}(\mathrm{x})$ & $0.4095(9)$ & 0.4128 & 0.4601 \\
$\mathrm{H}(\mathrm{y})$ & $0.0876(4)$ & 0.0907 & 0.0994 \\
$\mathrm{O}-\mathrm{H}[\AA]$ & $0.990(4)$ & 1.009 & 1.055 \\
$\left.\alpha{ }^{\circ}\right]$ & 12.1 & 11.4 & 9.5 \\
$B[\mathrm{GPa}]$ & $85-230^{1}$ & $147(2)^{3}, 149(6)^{4}$ & $360^{4}$ \\
$B^{\prime}$ & $5.8^{2}$ & $4.5(1)$ & \\
\hline
\end{tabular}

of the lattice parameters or cell angles) for each of the strain patterns. The stress tensors are then computed for each distorted structure, and assuming the linear relationship of Hooke's law, the elastic constants relating the applied strain to the resultant stress are obtained. On a conventional workstation, the computation of the equation of state and the elastic constants can be achieved within a few days.

\section{Results}

\subsection{Ambient pressure}

In an earlier study (Winkler et al., 1995), we have already shown that GGA-DFT calculations correctly reproduce the internal structural parameters. At that time, however, a full geometry relaxation was computationally too costly. Due to improvements in the computational approach, and the availability of faster workstations, full geometry optimizations of structures with a few tens of atoms are now straightforward. The results of such a full geometry optimization are shown in Table 1 and Table 3. There is good agreement between the calculated and experimentally determined structural parameters, and the model reproduces the small distortion of the $\mathrm{AlO}_{6}$ octahedra well. The electronic structure was interpreted by using a Mulliken population analysis. The corresponding results are given in Table 3 and 4 . The projection on a minimal basis set was successful as the 'spilling parameter', which describes the quality of the projection, was less than $1 \%$. The results obtained for the ambient pressure structure are compared to those given by Hill (1979). The "experimental" data have been obtained from an x-ray dif-

Table 2. Elastic constants of diaspore. The experimental $293 \mathrm{~K}$ values have been extrapolated to $0 \mathrm{~K}$ using the $T_{i j}$ of Haussühl (1993). The errors associated with the computed elastic constants and bulk moduli are derived from the fits relating the imposed strains to the computed stresses.

\begin{tabular}{llrrl|l}
\hline & $\begin{array}{l}\text { theo. } \\
\text { 0 GPa }\end{array}$ & $\begin{array}{l}\text { exp. } \\
\text { at 293 K }\end{array}$ & $\begin{array}{l}\text { exp. } \\
\text { at 0 K }\end{array}$ & diff & theo. \\
\hline$c_{11}$ & $237(8)$ & 250.7 & 259.5 & $-9 \%$ & $50 \mathrm{GPa}$ \\
$c_{22}$ & $347(3)$ & 336.8 & 350.6 & $-1 \%$ & 687 \\
$c_{33}$ & $400(2)$ & 394.2 & 405.8 & $-2 \%$ & 663 \\
$c_{12}$ & $73(20)$ & 66.7 & 71.2 & $+2 \%$ & 243 \\
$c_{13}$ & $46(15)$ & 41.7 & 40.2 & $+15 \%$ & 179 \\
$c_{23}$ & $103(1)$ & 95.3 & 99.2 & $+4 \%$ & 242 \\
$c_{44}$ & $129(2)$ & 127.1 & 131.6 & $-2 \%$ & 203 \\
$c_{55}$ & $124(1)$ & 96.9 & 101.1 & $+23 \%$ & 183 \\
$c_{66}$ & $128(1)$ & 116.2 & 122.0 & $+5 \%$ & 248 \\
\hline
\end{tabular}


fraction study in which the refinement included parameters for the core- and valence shell population and an additional parameter for the expansion or contraction of the valence shell density relative to that of a reference state. Obviously, with one exception, the theoretical data are in good agreement with the experimentally determined ones. The difference is for the charge of the $\mathrm{O}(2)$, which Hill (1979) found to be only about half of that of the $\mathrm{O}(1)$. The $\mathrm{O}(2)$ is bound to three $\mathrm{Al}$-atoms and one $\mathrm{H}$-atom, while the $\mathrm{O}(1)$ atom is only bound to three Al-atoms. Here, the Mulliken analysis yields only a slightly smaller net charge for the $\mathrm{O}(2)$ than for the $\mathrm{O}(1)$, similar to the results of a calculation based on 'extended Hückel molecular orbital' approach (Hill, 1979).

In contrast to the interpretation of the experimental deformation density (Hill, 1979) we find that some of the Al-O bonds have a rather large bond population and hence have an appreciably covalent character. This is the case for those Al-O bonds where the $z$-coordinate of the aluminum and oxygen atom differ. In diaspore, all atoms occupy positions with $z$ either 0.25 or 0.75 . While the bonds between atoms with equal $z$ are only weakly populated, those between atoms with different $z$ are appreciably covalent (Tab. 3).

As has been mentioned above, the experimentally determined values for the bulk modulus are in poor agreement with each other. From DFT calculations, the bulk modulus is usually obtained from $P-V$ data, similar to the approach used in $\mathrm{x}$-ray compression experiments. This will be discussed below. An alternative method is to compute the bulk modulus from the elastic constants (Nye, 1985). Hence we have computed the elastic constants according to the method of imposed strains as described above. These values describe the elas-

Table 3. Bond populations (in electrons) and bond distances, $r_{i j}$, in diaspore at 0 and $50 \mathrm{GPa}$. The experimental data are those given by Hill (1979). All atoms in diaspore are located at either $z=1 / 4$ or $z=3 / 4$. The primed atoms have a $z$ equal to the $\mathrm{Al}$ atom to which they are bound.

\begin{tabular}{l|l|ll|ll}
\hline atoms $i, j$ & exp. & \multicolumn{2}{|c|}{$0 \mathrm{GPa}$} & \multicolumn{2}{|c}{$50 \mathrm{GPa}$} \\
& $r_{i j}[\AA]$ & $r_{i j}[\AA \AA$ & bond & $r_{i j}[\AA ̊$ & bond \\
& & & pop. & & pop. \\
\hline $\mathrm{O}(2)-\mathrm{H}$ & $0.9886(8)$ & 1.009 & 0.58 & 1.055 & 0.54 \\
$\mathrm{O}(1)-\mathrm{H}$ & $1.6961(8)$ & 1.660 & 0.14 & 1.327 & 0.28 \\
$\mathrm{Al}-\mathrm{O}(1) \times 2$ & $1.8520(6)$ & 1.835 & 0.76 & 1.758 & 0.68 \\
$\mathrm{Al}-\mathrm{O}\left(1^{\prime}\right)$ & $1.8595(9)$ & 1.848 & 0.33 & 1.750 & 0.31 \\
$\mathrm{Al}-\mathrm{O}(2) \times 2$ & $1.9734(6)$ & 1.951 & 0.49 & 1.829 & 0.56 \\
$\mathrm{Al}-\mathrm{O}\left(2^{\prime}\right)$ & $1.9821(10)$ & 1.969 & 0.21 & 1.821 & 0.24 \\
\hline
\end{tabular}

tic properties of the ground state structure in the athermal limit. The corresponding experimental data (Haussühl, 1993) have been determined at ambient conditions. We have therefore used the observed thermoelastic constants, $T_{i j}$, defined as $T_{i j}=d \log c_{i j} / d T$ to extrapolate the experimental $c_{i j}$ to $0 \mathrm{~K}$. The $T_{i j}$ vanish as the temperature approaches $0 \mathrm{~K}$ and so the extrapolated $c_{i j}$ only approximate the $0 \mathrm{~K}$ values. The error introduced by the extrapolation is, however, insignificant with respect to the limited accuracy of the current approach. The results obtained here, given in Table 2 , generally agree with the experimental data to within $10 \%$, but $c_{13}$ and $c_{55}$ are overestimated by 15 and $23 \%$, respectively. The origin of these discrepancies is unknown. The relatively large errors associated with $c_{12}$ and $c_{13}$ are due to the use of more than one strain pattern in their derivation. These errors could be decreased by increasing the convergence parameters of the calculations, and by using smaller steps during the variation of the magnitude of the imposed strain patterns, but this would be computationally expensive without providing more insight. As $c_{55}$ corresponds to a volume-conserving distortion the bulk modulus $B=149$ (7) $\mathrm{GPa}$ computed from the $c_{i j}$ is still in very good agreement with the experimental data obtained from the elastic constants.

Table 4. Theoretical and experimentally determined (Hill, 1979) partial atomic charges in diaspore, $\mathrm{AlOOH}$. The first set of theoretical values are those derived from a plane wave/ultrasoft pseudopotential DFT study, with a subsequent projection on a minimal basis set at $0 \mathrm{GPa}$. The values given in the second line of each atom correspond to values obtained for a pressure of $50 \mathrm{GPa}$. The experimental values have been obtained from an $\mathrm{x}$-ray diffraction study in which the refinement included parameters for the coreand valence shell population and an additional parameter for the expansion or contraction of the valence shell density relative to that of a reference state. The results listed under EHMO are from an 'extended Hückel molecular orbital' calculation (Hill, 1979).

\begin{tabular}{lrrrrrrr}
\hline & $\mathrm{s}$ & $\mathrm{p}$ & total & charge & exp. & EHMO \\
\hline $\mathrm{Al}$ & 0.48 & 0.81 & 1.29 & +1.71 & $+1.47(26)$ & +2.09 \\
& 0.41 & 0.80 & 1.21 & +1.79 & & \\
$\mathrm{O}(1)$ & 1.84 & 5.27 & 7.11 & -1.11 & $-1.08(16)$ & -1.45 \\
& 1.82 & 5.28 & 7.11 & -1.11 & & \\
$\mathrm{O}(2)$ & 1.82 & 5.16 & 6.99 & -0.99 & $-0.59(13)$ & -1.13 \\
& 1.80 & 5.22 & 7.02 & -1.02 & & \\
$\mathrm{H}$ & 0.62 & 0.00 & 0.62 & +0.38 & $+0.20(5)$ & +0.39 \\
& 0.66 & 0.00 & 0.66 & +0.34 & & \\
\hline
\end{tabular}




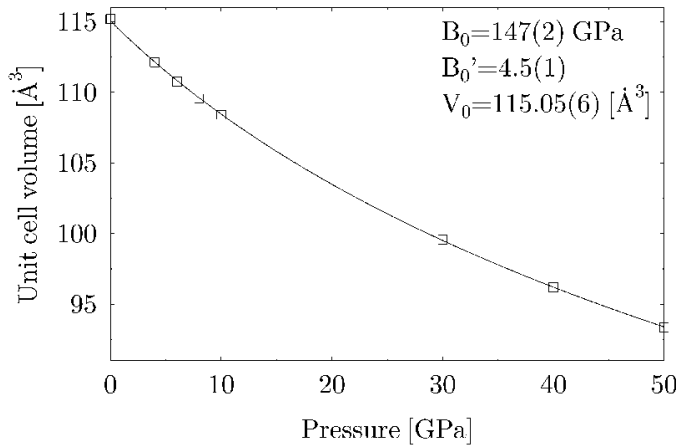

Fig. 1. Calculated pressure-volume relationship of diaspore. The line is a fit with a 3rd-order Birch-Murnaghan equation of state with the fit parameters shown in the figure.

\subsection{Pressure dependence}

For an analysis of pressure-induced changes of the bonds beyond what is presently possible with experimental methods, we relaxed the structure under applied pressures of up to $50 \mathrm{GPa}$. The computed volume-pressure relationship is shown in Fig. 1. From a 3rd-order Birch-Murnaghan equation of state fitted to the compression data we obtained $B=$ 147(2) $\mathrm{GPa}$ and $B^{\prime}=4.5(1)$. The errors associated with these values are those from the least-square fit to the eight $P-V$ data points. The first value is in very good agreement with the data derived from the experimentally determined and theoretically obtained elastic constants. All experimental studies and the results obtained here agree in that the $a$-axis is the most compressible in diaspore. At zero pressure, the $c$-axis is stiffest according to the computed and experimentally determined elastic constants. This changes on increasing pressure, as the computed pressure-induced changes of the lattice constants imply that at high pressures the $b$-axis becomes stiffer than the $c$-axis (Table 1).

From our calculations, we derive a smooth decrease of the $\mathrm{H}$...O(1) distance from $1.66 \AA$ to 1.33 $\AA$ over the pressure interval from 0 to $50 \mathrm{GPa}$. The $\mathrm{O}(1)-\mathrm{O}(2)-\mathrm{H}$ angle decreases smoothly from $11.4^{\circ}$ at $0 \mathrm{GPa}$ to $9.5^{\circ}$ at $50 \mathrm{GPa}$. Concomitantly, the $\mathrm{O}(2)-\mathrm{H}$ distance increases slightly by $0.05 \AA$, implying a pressure-induced strengthening of the $\mathrm{H}$...O(1) hydrogen bond (Fig. 2). This is also obvious from the two-fold increase in the bond population of the hydrogen bond (Table 3 ). The changes in the bond population as a function of bond distances are shown in Fig. 3, which clearly shows that the change in the population of the $\mathrm{H}$...O $\mathrm{O}(1)$ bond is the

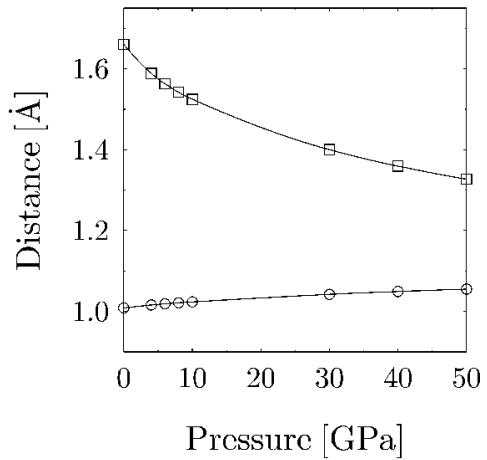

Fig. 2. Pressure-induced changes in the $\mathrm{O}(2)-\mathrm{H}$ distance (open circles) and the $\mathrm{H} . . . \mathrm{O}(1)$ distance (open squares). This behavior implies a pressure-induced strengthening of the H...O(1) hydrogen bond.

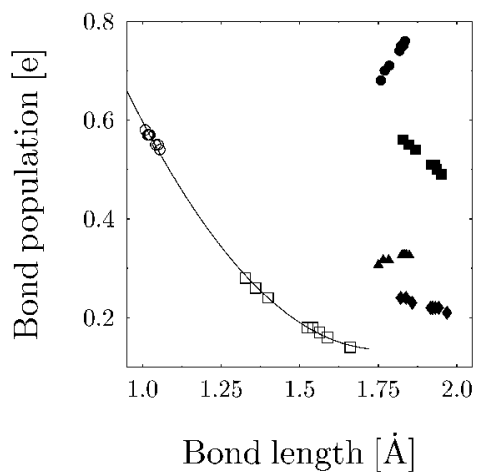

Fig. 3. Calculated dependence of the bond population of bonds in diaspore as a function of bond length. The open symbols indicate the $\mathrm{O}-\mathrm{H}$ (open circle) and $\mathrm{H}$...O (open square) bonds. The line is a guide to the eye, indicating that the change of the population of both the $\mathrm{O}-\mathrm{H}$ and the $\mathrm{H}$... $\mathrm{O}$ bond can be described with a single function. The filled symbols represent the $\mathrm{Al}-\mathrm{O}(1)$ bond (filled circle), the $\mathrm{Al}(1$ ') bond (filled triangle), the $\mathrm{Al}-\mathrm{O}(2)$ bond (filled squares) and the $\mathrm{Al}-\mathrm{O}(2$ ') bond (filled diamond). Primed oxygen atoms have the same $z$-coordinate as the Al-atom to which they are bound. The bond distances were varied by applying pressure to the structure. Clearly, the bond population of the Al-O bonds is not a simple function of distance.

largest change we observe. The pressure-induced changes of the bond populations also clearly indicate that the Al-O bonds in diaspore are rather different (Table 3). While for individual bonds there is a clear dependence of the bond population on the bond distance, there is no correlation between the pressure-induced change of the bond lengths and changes of the bond populations of different Al-O bonds. The origin of the different behaviour of the $\mathrm{Al}-\mathrm{O}(1)$ bonds with respect to the $\mathrm{Al}-\mathrm{O}(2)$ bonds is 
probably a direct consequence of the increasing strength of the hydrogen bonds. As the H...O(1) bond gets stronger on compression, increasingly more charge is transferred from the $\mathrm{Al}-\mathrm{O}(1)$ bonds to the $\mathrm{H} . . . \mathrm{O}(1)$ bond, causing a decrease of the $\mathrm{Al}-\mathrm{O}(1)$ bond population as the bonds get shorter.

\section{Discussion}

The consistency between experimentally determined elastic constants, elastic constants obtained theoretically and the bulk modulus obtained from theoretical $P-V$ data indicates that the most reliable value for the bulk modulus of diaspore is $B \approx 148$ $\mathrm{GPa}$, with an error of a few percent. The calculations predict a $B^{\prime}=4.5(1)$, which, based on the reliability of the other computed data, we judge to be more reliable than the value $B^{\prime}=5.8$ used earlier (Fasshauer et al., 1998). Hence, phase equilibria calculated from internally consistent databases relying on the earlier values obtained from x-ray diffraction experiments should be reevaluated.

The Mulliken population analysis of the ground state structure indicates that some of the Al-O bonds have an appreciable amount of covalent character, in agreement with conventional crystal chemical concepts. That the deformation map obtained experimentally did not show a charge accumulation between these atoms is probably due to experimental problems, such as extinction or the limited resolution of the experimental data set. Also, the suspiciously small partial charge found experimentally on the $\mathrm{O}(2)$ atom seems to be unreliable, and the value obtained here is very likely more consistent with the other partial charges.

Most pressure-induced changes to the interatomic bonds seem to be related to the increase in the strength of the hydrogen-bond on compression. The current calculations of the pressure-induced changes in the $\mathrm{O}-\mathrm{H}$...O group imply that as the strength of the H...O interaction increases, the strength of the $\mathrm{O}-\mathrm{H}$ bond decreases, which would imply that there is a continuous decrease of the $\mathrm{O}-\mathrm{H}$ stretching frequency. In goethite, $\boldsymbol{\alpha}-\mathrm{FeOOH}$, which is isostructural to diaspore, Williams \& Guenther (1996) observed two stretching vibrations, one which decreased in frequency over the pressure interval of $0-25 \mathrm{GPa}$, while the other increased. This peculiar behavior was thought to be due to the influence of "cation-hydrogen repulsive interactions". However, the quality of the spectra obtained in the $\mathrm{OH}$-stretching region was rather poor. The current calculations imply that at least for diaspore the compression mechanism is much simpler. This could be verified by rather straightforward highpressure infrared spectroscopic experiments. For this, it would be preferable to use appropriately cut single crystals, as this would probably lead to spectra of higher quality (Winkler et al., 1989).

From a more general perspective, we believe that the present study has shown that quantum mechanical models can be used to check inconsistent experimental data. The recently developed population analysis of plane-wave results has been applied here successfully to a low-symmetry crystal structure. As the model is parameter-free and can be applied to structures with up to a hundred atoms without incurring excessive computational costs we expect that this approach will become increasingly used to understand the compression mechanisms of minerals.

Acknowledgments: This study was partially funded by the German Science Foundation (Grant Wi1232/10) and a grant from the Fonds der Chemischen Industrie. We would like to thank Jürgen Schreuer (ETH Zürich) for the organization of a workshop which initiated this study and Karsten Knorr (Kiel) for helpful discussions.

\section{References}

Bachelet, G. B., Hamann, D. R., Schlüter, M. (1982) : Pseudopotentials that work: from $\mathrm{H}$ to Pu. Phys. Rev. B, 26, 4199-4228.

Busing, W. R. \& Levy, H. A. (1958) : A single crystal neutron diffraction study of diaspore, $\mathrm{AlO}(\mathrm{OH})$. Acta Cryst, 11, 798-803.

Fasshauer, D. W, Chatterjee, N. D., Cemic, L. (1998): A thermodynamic analysis of the system $\mathrm{LiAlSiO}_{4}-\mathrm{NaAl}-$ $\mathrm{SiO}_{4}-\mathrm{Al}_{2} \mathrm{O}_{3}-\mathrm{SiO}_{2}-\mathrm{H}_{2} \mathrm{O}$ based on new heat capacity, thermal expansion, and compressibility data for selected phases. Contrib. Mineral. Petrol., 133, 186-198.

Fockenberg, T., Wunder, B., Grevel, K.-D., Burchard, M. (1996): The equilibrium of diaspore-corundum at high pressures. Eur. Mineral., 8, 1293-1299.

Goniakowski, J., Holender, J. M., Kantorovich, L. N., Gillan, M. J., White, J. A. (1996): Influence of gradient corrections on the bulk and surface properties of $\mathrm{TiO}_{2}$ and $\mathrm{SnO}_{2}$. Phys. Rev. B, 53, 957-960.

Hamann, D. R. (1996): Generalized gradient theory for silica phase transitions. Phys. Rev. Lett., 76, 660-663.

Hammer, B., Jacobsen, K. W., Norskov, J. K. (1993): Role of nonlocal exchange-correlation in activated adsorption. Phys. Rev. Lett., 70, 3971-3974.

Haussühl, S. (1993): Thermoelastic properties of beryl, topaz, diaspore, sanidine and periclase. Zeit. Kristall., 204, 67-76. 
Hill, R. J. (1979): Crystal structure refinement and electron density distribution in diaspore. Phys. Chem. Minerals, 5, 179-200.

Hohenberg, P. \& Kohn, W. (1964): Inhomogeneous electron gas. Phys. Rev., 136, B864-B871.

Holland, T. J. B., Redfern, S. A. T., Pawley, A. R. (1996): Volume behaviour of hydrous minerals at high pressure and temperature: II. Compressibilities of lawsonite, clinozoisite and epidote. Am. Mineral., 81, 341-348.

Jones, R. O. \& Gunnarsson, O. (1989): The density functional formalism, its applications and prospects. Rev. Modern Physics, 61, 689-746.

Kleinman, L. \& Bylander, D. M. (1982): Efficacious form for model pseudopotentials. Phys. Rev. Lett., 48, 14251428.

Kohn, W. \& Sham, L. J. (1965): Self-consistent equations including exchange and correlation effects. Phys. Rev., 140, A1133-A1138.

Kresse, G. \& Hafner, J. (1994): Norm-conserving and ultrasoft pseudopotentials for first-row and transition elements. J. Physics: Condensed Matter, 6, 8245-8257.

Kryachko, E. S. \& Ludena, E. V. (1990): Energy density functional theory of many electron systems, volume 4 of Understanding Chemical Reactivity. Kluwer Academic Publishers, Dordrecht.

Leung, T. C., Chan, C. T., Harmon, B. N. (1991): Groundstate properties of $\mathrm{Fe}, \mathrm{Co}, \mathrm{Ni}$ and their monoxides: results of the generalized gradient approximation. Phys. Rev. B, 44, 2923-2927.

Mao, H.-K., Shu, J., Hu, J., Hemley, R. J. (1994): High pressure $\mathrm{x}$-ray diffraction study of diaspore. Solid State Comm., 90, 497-500.

Milman, V., Winkler, B., White, J. A., Pickard, C. J., Payne, M. C., Akhmatskaya, E. V., Nobes, R. H. (2000): Electronic structure, properties, and phase stabilities of inorganic crystals: a pseudopotential plane-wave study. Intern. Quantum Chemistry, 77, 895-910.

Monkhorst, H. J. \& Pack, J. D. (1976): Special points for Brillouin-zone integration. Phys. Rev. B, 13, 51885192.

MSI 1998 (1998): CASTEP User Guide. Molecular Simulations Inc., San Diego, CA.

Nye, J. F. (1985): Physical Properties of Crystals. Oxford Science Publications, Oxford.

Parr, R. G. \& Yang, W. (1989): Density-functional theory of atoms and molecules. Oxford University Press.

Pawley, A. R., Redfern, S. A. T., Holland, T. J. B. (1996): Volume behavior of hydrous minerals at high pressure and temperature: I. Thermal expansion of lawsonite, zoisite, clinozoisite, and diaspore. Am. Mineral., 81, 341-348.

Payne, M. C., Teter, M. P., Allan, D. C., Arias, T. A., Johannopoulos, J. D. (1992): Iterative minimisation techniques for $\mathrm{ab}$ initio total energy calculations - molecular dynamics and conjugate gradients. Rev. Modern Physics, 64, 1045-1097.

Perdew, J. P., Burke, K., Ernzerhof, M. (1996): Generalized gradient approximation made simple. Phys. Rev. Lett., 77, 3865-3868.

Ruoff, A. L. \& Vanderborgh, C. A. (1991): Hydrogen reduction of ruby at high pressure: implication for claims of metallic hydrogen. Phys. Rev. Lett., 66, 754-757.

Ryskin, Y. I. (1974): The vibrations of protons in minerals: hydroxyl, water and ammonium. In Farmer, V., ed., The infrared spectra of minerals, chapter 9, p. 137-182. Mineralogical Society, London.

Sanchez-Portal, D., Artacho, E., Soler, J. M. (1996): Analysis of atomic orbital basis sets from the projection of plane-wave results. J. Physics: Condensed Matter, 8, 3859-3880.

Segall, M. D., Shah, R., Pickard, C. J., Payne, M. C. (1996): Population analysis of plane-wave electronic structure calculations of bulk materials. Phys. Rev. B, 54, 1631716320.

Vanderbilt, D. (1990): Soft self-consistent pseudopotentials in a generalized eigenvalue formalism. Phys. Rev. $B, \mathbf{4 1}, 7892-7895$.

Williams, Q. \& Guenther, L. (1996): Pressure-induced changes in the bonding and orientation of hydrogen in FeOOH-goethite. Solid State Comm., 100, 105-109.

Winkler, B., Langer, K., Johannsen, P. G. (1989): The influence of pressure on the $\mathrm{OH}$ valence vibration of zoisite. Phys. Chem. Minerals, 16, 668-671.

Winkler, B., Milman, V., Hennion, B., Lee, M. H., Lin, J. S. (1995): Ab initio total energy study of brucite, diaspore and hypothetical hydrous wadsleyite. Phys. Chem. Minerals, 22, 461-467.

Xu, J.-A., Hu, J., Ming, L.-C., Huang, E., Xie, H. (1994): The compression of diaspore, $\mathrm{AlO}(\mathrm{OH})$ at room temperature up to $27 \mathrm{GPa}$. Geophys. Research Lett., 21, $161-164$.

Received 20 July 2000

Accepted 10 November 2000 